\title{
Collaborative Overtaking of Multi-Vehicle Systems in Dynamic Environments: A Distributed Artificial Potential Field Approach
}

DOI:

10.1109/ICAR53236.2021.9659390

\section{Document Version}

Accepted author manuscript

Link to publication record in Manchester Research Explorer

Citation for published version (APA):

Xie, S., Hu, J., Ding, Z., \& Arvin, F. (2021). Collaborative Overtaking of Multi-Vehicle Systems in Dynamic Environments: A Distributed Artificial Potential Field Approach. In 2021 20th International Conference on Advanced Robotics, ICAR 2021 (pp. 873-878). (2021 20th International Conference on Advanced Robotics, ICAR 2021). https://doi.org/10.1109/ICAR53236.2021.9659390

\section{Published in:}

2021 20th International Conference on Advanced Robotics, ICAR 2021

\section{Citing this paper}

Please note that where the full-text provided on Manchester Research Explorer is the Author Accepted Manuscript or Proof version this may differ from the final Published version. If citing, it is advised that you check and use the publisher's definitive version.

\section{General rights}

Copyright and moral rights for the publications made accessible in the Research Explorer are retained by the authors and/or other copyright owners and it is a condition of accessing publications that users recognise and abide by the legal requirements associated with these rights.

\section{Takedown policy}

If you believe that this document breaches copyright please refer to the University of Manchester's Takedown Procedures [http://man.ac.uk/04Y6Bo] or contact uml.scholarlycommunications@manchester.ac.uk providing relevant details, so we can investigate your claim.

\section{OPEN ACCESS}




\title{
Collaborative Overtaking of Multi-Vehicle Systems in Dynamic Environments: A Distributed Artificial Potential Field Approach
}

\author{
Songtao Xie, Student Member, IEEE, Junyan Hu, Member, IEEE, Zhengtao Ding, Senior Member, IEEE, \\ and Farshad Arvin, Senior Member, IEEE
}

\begin{abstract}
Multi-vehicle autonomous driving has attracted significant attention from academia and industries due to its ability to improve traffic efficiency and enhance the safety of drivers and passengers. In this paper, a distributed motion planning strategy based on the artificial potential field method is proposed to achieve overtaking of the autonomous vehicle fleet in dynamic environments. Firstly, a dynamic target tracking control protocol is constructed for an autonomous vehicle fleet using a modified artificial potential field. The target tracking control protocol overcomes the disadvantage of the traditional artificial potential field method (i.e., the dynamic target cannot be reached). Besides, a distributed obstacle avoidance control protocol is also designed to avoid potential collisions during the overtaking process. Finally, simulation experiments are performed to verify the feasibility and effectiveness of the proposed algorithm.
\end{abstract}

\section{INTRODUCTION}

The globally increasing number of cars has already brought many critical issues [1], such as traffic congestion, pollution, and road safety. Autonomous driving is considered as an emerging technology that may effectively overcome these challenges [2]. In addition to reducing human-caused traffic accidents, autonomous driving technology could have a positive effect in terms of better fuel economy [3], reduced pollution, protected public health [4], and improved traffic efficiency [5].

There are many studies on AVs' perception, decisionmaking, motion planning, motion control, and traffic scheduling problems [6]-[10]. The research studies on self-driving technology mainly focused on single-vehicle systems in the past decade. In recent years, increasing attention have been paid to multi-vehicle systems [11]-[13]. As described in [14], the emergence of connected autonomous vehicles $(\mathrm{AVs})$ can significantly improve the efficiency of the traffic. By information sharing and collaborative sensing (as portrayed in Fig. 1), joint operation of multi-vehicle systems promotes the cooperation among intelligent vehicles, thus improving the overall driving quality and safety [15]-[17].

Collaborative overtaking is a very important practical issue in multi-vehicle systems. To achieve this objective, some preliminary results have been developed by researchers. Hegedús et al. [18] proposed an advanced graph-based optimal solution for overtaking scenarios of multiple vehicles. On this basis, Nemeth et al. [19] proposed another method of an automatic vehicle overtaking based on the model predictive control, where the graph optimization algorithm provides

The authors are with the Department of Electrical and Electronic Engineering, University of Manchester, Manchester, M13 9PL, U.K. (e-mail: \{songtao.xie, junyan.hu, zhengtao.ding, farshad.arvin\}@manchester.ac.uk)

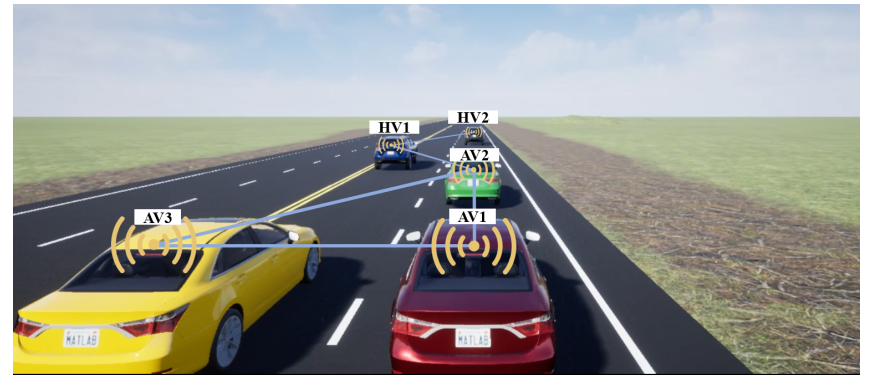

Fig. 1. Autonomous vehicles and human-driven vehicles are connected by V2V communication on highway system. The AV and HV denote, respectively, the autonomous vehicle and human-driven vehicle.

the path of obstacle avoidance and overtaking. In [20], a unified approach for cooperative path-planning based on a nonlinear model predictive control was proposed. Consider that the trajectory prediction of the human driver model was integrated into the framework, the behaviors of the other agents were affected by the human-driven vehicles [21]. In [22], a distributed control method for coordinating multiple vehicles in the framework of an automated valet parking system was introduced. The main limitation is to rely on traffic infrastructure, which poses a considerable challenge to the current traffic facilities. Karlsson et al. [23] proposed an integrated route and motion planning approach that achieves scalability concerning the number of cars. However, in this work, vehicles other than navigators cannot interact with other vehicle members during the overtaking.

Motivated by the aforementioned progress and challenges in the area of multi-vehicle overtaking, in this paper, a novel distributed motion planning approach for multi-vehicle collaborative overtaking is proposed. The method presented in this paper is to transform the overtaking of the automatic driving fleet into multiple dynamic target tracking problems by assigning a virtual dynamic target for each team member. Based on an improved artificial potential field, we construct a dynamic target tracking control protocol for an AV fleet. Simulation studies are performed to verify the feasibility and effectiveness of the proposed distributed motion planning method. The main contributions of this paper are listed as follows:

- A distributed motion planning algorithm based on the modified artificial potential field is proposed. To the best of the authors' knowledge, such a novel coordination design has not been developed in the relevant studies.

- A distributed obstacle avoidance control protocol based 
on the artificial potential field is proposed, which ensures the AV fleet avoid collisions when execute overtaking.

- The effectiveness of the proposed strategy for use in AV overtaking scenario is validated by a realistic simulator, Unreal Engine. Hence, the proposed algorithm can then be possibly applied to the existing AVs' software and hardware architecture.

\section{PRELIMINARIES AND PROBLEM STATEMENTS}

\section{A. Control objectives}

In this paper, we decompose the overtaking scenario of a multi-vehicle system into a multi-target tracking problem. To achieve overtaking, a dynamic virtual target is set up for each vehicle. Hence, the control objectives are given as follows:

- The vehicle needs to complete the tracking of dynamic virtual objects as follows,

$$
\begin{aligned}
& \lim _{t \rightarrow \infty} d\left(P_{i}, P_{g}\right)(t)=0, \\
& \lim _{t \rightarrow \infty} d\left(V_{i}, V_{g}\right)(t)=0, \\
& \lim _{t \rightarrow \infty} d\left(a_{i}, a_{g}\right)(t)=0,
\end{aligned}
$$

where $d\left(P_{i}, P_{g}\right), d\left(V_{i}, V_{g}\right)$, and $d\left(a_{i}, a_{g}\right)$ are the distance difference, velocity difference, and acceleration difference between the vehicle $i$ and virtual target $g$, respectively.

- To avoid collisions between interacting vehicles and human-driven vehicles, the following equation must be satisfied

$$
\forall i, j \in, i \neq j:\left\|X_{i}-X_{j}\right\|>D
$$

where $D$ is defined as the obstacle avoidance area of the vehicle, within which other vehicles are not allowed to approach. $X_{i}$ and $X_{j}$ are, respectively, the position of the vehicle $i$ and vehicle $j$.

\section{B. Communication topology}

Define a security area $\rho$ where each vehicle seeks to keep clear of other multi-vehicle system members and humandriven vehicles. Define the collision neighborhood for each vehicle $i$ as $N_{i}^{c}=\left\{j: d_{i j} \leq \rho\right\}$.

We assume that the multi-vehicle system contains $n$ AVs and $m$ human-driven vehicles (HVs) in the environment. Describe the multi-vehicle system by a mixed graph $G(N, \varepsilon(t), A(t))$, where $N$ and $\varepsilon(t) \in n \times(n+m)$ are the set of nodes and and set of arcs, respectively. $A(t)=\left[a_{i j}\right] \in$ $\mathbb{R}^{n \times(n+m)}$, if other vehicles enter the collision neighborhood of the vehicle $i,(j, i) \in \varepsilon(t)$ and $a_{i j}=1$, otherwise, $a_{i j}=0$, where $i$ is the incoming neighbor of $j$, and $j$ is the outgoing neighbor of $i$. Fig. 2 describes the communication relationship between agent $i$ and its neighbors. The agent $i\left(i^{t h}\right.$ AV) will establish bidirectional communication with other autonomous vehicles and unidirectional communication with human-driven vehicles within the security area of agent $i$. It is clear that in this paper, graph $G$ is a dynamic mixed graph that changes over time. Besides, we set up a one-to-one

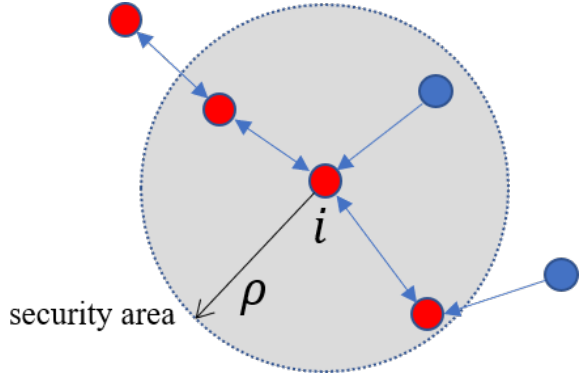

Fig. 2. The communication relationship between vehicle $i$ and other vehicles. The red circles represent the autonomous vehicle, blue circles represent the human-driven vehicles.

communication protocol between the vehicle and the virtual target. When communication is established between vehicles, vehicle $i$ can receive the position, velocity and acceleration information of vehicle $j$ and virtual target $g$.

\section{Basic assumptions}

To formulate the problem, we have the following assumptions:

- All vehicles are equipped with Vehicle-to-Vehicle (V2V) communication technology to achieve information exchange.

- In the process of overtaking with a multi-vehicle system, both right side overtaking and left side overtaking are allowed.

- The dynamics of the AVs can be described by the following particle system.

$$
\begin{aligned}
\dot{X}_{i} & =V_{i}, \\
\dot{V}_{i} & =a_{i}, \\
m_{i} \dot{a}_{i} & =F_{i},
\end{aligned}
$$

where $F_{i}$ is the control input of the vehicle $i . m_{i}$ is the mass of vehicle $i . V_{i}$ and $a_{i}$ denote the velocity and acceleration of vehicle $i$, respectively.

\section{CONTROL PROTOCOL DESIGN}

In this section, we develop a novel distributed control protocol based on artificial potential field method. Different from the traditional artificial potential field method which is suitable for tracking static targets [24], in this work, we modify the traditional artificial potential field such that it can be used to track dynamic targets. In addition, the tracking algorithm is improved to solve the problem of unreachable target in the artificial potential field method. For the problem of vehicle obstacle avoidance, we set up a distributed repulsive artificial potential field to realize this objective.

\section{A. Target tracking control protocol}

Between the $i^{t h} \mathrm{AV}$ and the $i^{t h}$ virtual goal node, we define the following attractive potential field. 


$$
\begin{aligned}
U_{a t t}(P, V, a)= & \frac{1}{2} K_{P} d^{2}\left(P_{i}, P_{g}\right)+\frac{1}{2} K_{v} d^{2}\left(V_{i}, V_{g}\right) \\
& +\frac{1}{2} K_{a} d^{2}\left(a_{i}, a_{g}\right),
\end{aligned}
$$

where $K_{p}>0, K_{v}>0$ and $K_{a}>0$ are position, velocity, and acceleration gain coefficients respectively.

The attractive force produced by the attractive potential field is:

$$
\begin{aligned}
F_{a t t}(i)= & -\nabla U_{a t t}(P, V, a) \\
= & -\frac{\partial U_{a t t}(P, V, a)}{\partial P}-\frac{\partial U_{a t t}(P, V, a)}{\partial V} \\
& -\frac{\partial U_{a t t}(P, V, a)}{\partial a} \\
= & -K_{p}\left(X_{i}-X_{g}\right)-K_{v}\left(\dot{X}_{i}-\dot{X}_{g}\right) \\
& -K_{a}\left(\ddot{X}_{i}-\ddot{X}_{g}\right) .
\end{aligned}
$$

The tracking control protocol is modified to obtain the following control input:

$$
\begin{aligned}
F_{\text {att }}(i)= & -K_{p}\left(X_{i}-X_{g}-\frac{m \dddot{X}_{g}}{K_{p}}\right)-K_{v}\left(\dot{X}_{i}-\dot{X}_{g}\right) \\
& -K_{a}\left(\ddot{X}_{i}-\ddot{X}_{g}\right) .
\end{aligned}
$$

We define the difference between the position of the $i^{t h}$ vehicle fleet member and the $i^{t h}$ virtual dynamic target as the control object:

$$
\left\{\begin{array}{l}
e=X_{i}-X_{g} \\
\dot{e}=\dot{X}_{i}-\dot{X}_{g} \\
\ddot{e}=\ddot{X}_{i}-\ddot{X}_{g} \\
\dddot{e}=\dddot{X}_{i}-\dddot{X}_{g}
\end{array}\right.
$$

Consider the following particle dynamical system

$$
\begin{aligned}
\dddot{X}_{i}= & \frac{1}{m}\left[-K_{p}\left(X_{i}-X_{g}-\frac{m \dddot{X}_{g}}{K_{p}}\right)-K_{v}\left(\dot{X}_{i}-\dot{X}_{g}\right)\right. \\
& \left.-K_{a}\left(\ddot{X}_{i}-\ddot{X}_{g}\right)\right] .
\end{aligned}
$$

Substitute Eq. (9) into Eq. (10), we have

$$
\dddot{e}=\frac{1}{m}\left[-K_{p}\left(e-\frac{m \dddot{X}_{g}}{K_{p}}\right)-K_{v}(\dot{e})-K_{a}(\ddot{e})\right]-\dddot{X}_{g},
$$

and its steady state value is obtained as follows,

$$
E=\{\ddot{e}, \dot{e}, e \mid e=\ddot{e}=\dot{e}=0\} .
$$

Such a steady state set of values means that the position, speed and acceleration parameters of the autonomous driving vehicle can converge to the virtual target. When every vehicle in the multi-vehicle system enters the steady state, it means that the control objectives of tracking are completed.

\section{B. Obstacle avoidance control protocol}

The repulsion field is created between the $i^{\text {th }} \mathrm{AV}$ and the $j^{\text {th }}$ human-driven vehicle or the AV fleet member as shown in Eq. (7), where $\eta_{p}>$ and $\eta_{v}>0$ are the position repulsive force gain and the velocity repulsive force gain, respectively. Define that the gradient of repulsive potential fields is the repulsive force, then it can be described by Eq. (8) as shown at the bottom of the page.

It can be obtained that the obstacle avoidance control input of the vehicle $i$ is:

$$
F_{r e p}(i)=\sum_{j \in N_{i}^{c}} a_{i j} F_{r e p}(i j) .
$$

This control protocol is used to achieve the control objective of obstacle avoidance.

Hence, we obtain a target tracking control input with the ability of obstacle avoidance

$$
F(i)=\sum_{j \in N_{i}^{c}} a_{i j} F_{r e p}(i j)+F_{a t t}(i),
$$

which can be directly applied to the vehicle dynamical model Eq. (3).

\section{RESUltS AND ANALYSES}

In this section, a simulation case study is conducted to verify the effectiveness of the proposed algorithm. Fig. 3 describes a typical overtaking scenario of an AV fleet. In this scenario, the road is a divided highway with four lanes. A fleet of three autonomous cars need to overtake two human-driven vehicles in front of them. Unreal Engine and Matlab are used in the simulation experiments. Unreal Engine is a powerful game physics engine that can be used to

$$
\begin{aligned}
U_{\text {rep }(\mathrm{ij})}= & \left\{\begin{array}{l}
\frac{1}{2} \eta_{p}\left(\frac{1}{d\left(P_{j}, P_{i}\right)}-\frac{1}{\rho}\right)^{2} d\left(P_{g}, P_{i}\right)+\eta_{v} d\left(V_{j}, V_{i}\right), \quad \text { if } d\left(P_{j}, P_{i}\right) \leq \rho \text { and } d\left(V_{j}, V_{i}\right) \cdot d\left(P_{j}, P_{i}\right)>0 ; \\
\frac{1}{2} \eta_{p}\left(\frac{1}{d\left(P_{j}, P_{i}\right)}-\frac{1}{\rho}\right)^{2} d\left(P_{g}, P_{i}\right), \quad \text { if } d\left(P_{j}, P_{i}\right) \leq \rho \text { and } d\left(V_{j}, V_{i}\right) \cdot d\left(P_{j}, P_{i}\right) \leq 0 ; \\
0, \quad \text { if } d\left(P_{j}, P_{i}\right)>\rho .
\end{array}\right. \\
F_{\text {rep }}(i j) & =-\nabla U_{r e p(i j)}(P, V) \\
& =\left\{\begin{array}{l}
\eta_{p}\left(\frac{1}{X_{i}-X_{j}}-\frac{1}{\rho}\right) \frac{X_{i}-X_{g}}{X_{i}-X_{j}}+\frac{1}{2} \eta_{p}\left(\frac{1}{X_{i}-X_{j}}-\frac{1}{\rho}\right)^{2}+\eta_{v}, \quad \text { if } d\left(V_{j}, V_{i}\right) \cdot d\left(P_{j}, P_{i}\right)>0 ; \\
\eta_{p}\left(\frac{1}{X_{i}-X_{j}}-\frac{1}{\rho}\right) \frac{X_{i}-X_{g}}{X_{i}-X_{j}}+\frac{1}{2} \eta_{p}\left(\frac{1}{X_{i}-X_{j}}-\frac{1}{\rho}\right)^{2}, \text { if } d\left(V_{j}, V_{i}\right) \cdot d\left(P_{j}, P_{i}\right) \leq 0 .
\end{array}\right.
\end{aligned}
$$




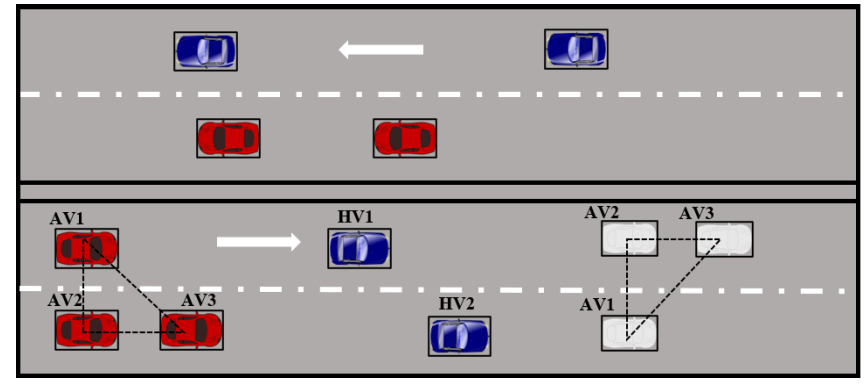

Fig. 3. A typical overtaking scenario of autonomous vehicle fleet. The red cars AV1,AV2,AV3 are three autonomous vehicles; blue cars HV1, HV2 are human-driven vehicles; grey cars represent the target position of AV fleet.

build very realistic autopilot scenarios that are close to realworld driving environments. Matlab is used for algorithm development. Experimental parameters are shown as Table I.

TABLE I

THE SIMULATION PARAMETERS IN CASE STUDY

\begin{tabular}{ll}
\hline Simulation parameters & Value \\
\hline Initial position of AV1 & $(0,-2.875) \mathrm{m}$ \\
Initial position of AV2 & $(10,-2.875) \mathrm{m}$ \\
Initial position of AV3 & $(0,0.875) \mathrm{m}$ \\
Initial position of HV1 & $(20,1.5) \mathrm{m}$ \\
Initial position of HV2 & $(40,-3) \mathrm{m}$ \\
Initial velocity of AV1 & $2 \mathrm{~m} / \mathrm{s}$ \\
Initial velocity of AV2 & $5 \mathrm{~m} / \mathrm{s}$ \\
Initial velocity of AV3 & $8 \mathrm{~m} / \mathrm{s}$ \\
Initial velocity of HV1 & $10 \mathrm{~m} / \mathrm{s}$ \\
Initial velocity of HV2 & $10 \mathrm{~m} / \mathrm{s}$ \\
Initial acceleration of AV1 & $0 \mathrm{~m} / \mathrm{s}^{2}$ \\
Initial acceleration of AV2 & $0 \mathrm{~m} / \mathrm{s}^{2}$ \\
Initial acceleration of AV3 & $0 \mathrm{~m} / \mathrm{s}^{2}$ \\
Acceleration of HV1 & $0.1 \mathrm{~m} / \mathrm{s}^{2}$ \\
Acceleration of HV2 & $0.1 \mathrm{~m} / \mathrm{s}^{2}$ \\
Initial position of Goal1 & $(60,1.375) \mathrm{m}$ \\
Initial position of Goal2 & $(70,1.375) \mathrm{m}$ \\
Initial position of Goal3 & $(70,-2.75) \mathrm{m}$ \\
Initial velocity of Goal1, Goal2, Goal3 & $10 \mathrm{~m} / \mathrm{s}$ \\
Acceleration of Goal1, Goal2, Goal3 & $0.1 \mathrm{~m} / \mathrm{s}^{2}$ \\
Attractive force gain $\left(K_{p}, K_{V}, K_{a}\right)$ & $(220,1000,100)$ \\
Repulsive force gain of vehicles $\left(\eta_{p}, \eta_{V}\right)$ & $(100,200)$ \\
Repulsive force gain of road boundary & 300 \\
Mass of vehicle & $1000 \mathrm{~kg}$ \\
Sampling time & $0.1 \mathrm{~s}$ \\
Maximum velocity in longitudinal & $33 \mathrm{~m} / \mathrm{s}$ \\
Maximum velocity in lateral & $5 \mathrm{~m} / \mathrm{s}$ \\
The jerk of human-driven vehicles & $0 \mathrm{~m} / \mathrm{s}^{3}$ \\
\hline &
\end{tabular}

The simulation result in Fig. 4 clearly shows the trajectories of $\mathrm{AV}$ fleet and the human-driven vehicles. In the process of overtaking, cooperative obstacle avoidance behaviors can be performed by the team members. The AV fleet finally completes the overtaking and each AV fleet member reaches the desired position. In addition, it can be seen from the simulation results that in the early stage of the whole movement process, the AV fleet members mainly complete a series of complex actions such as lane change, cooperative obstacle avoidance and overtaking. In the later stage, the AV fleet members mainly complete the tracking

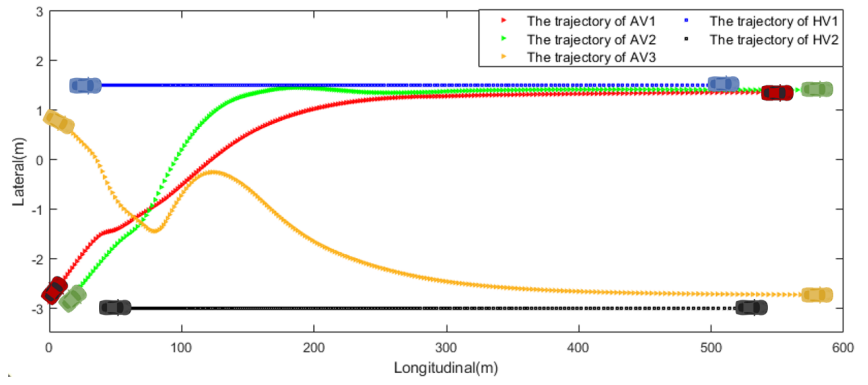

Fig. 4. Overtaking trajectories of the autonomous vehicle fleet.

of the dynamic virtual targets.

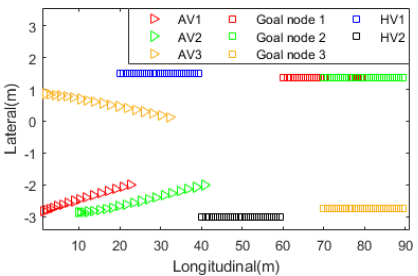

(a) $0-2 \mathrm{~s}$

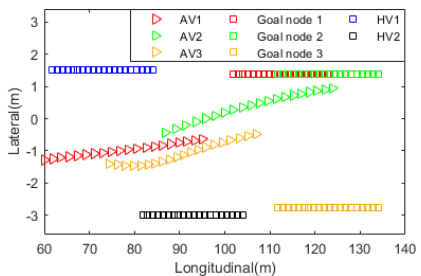

(c) $4-6 \mathrm{~s}$

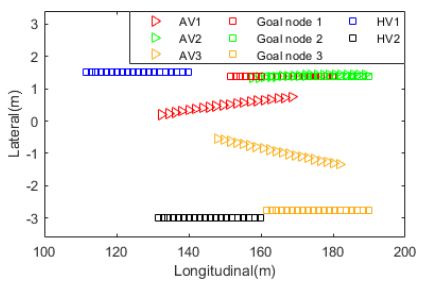

(e) $8-10 \mathrm{~s}$

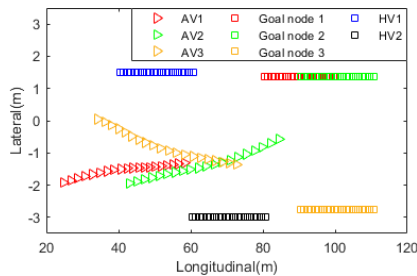

(b) $2-4 \mathrm{~s}$

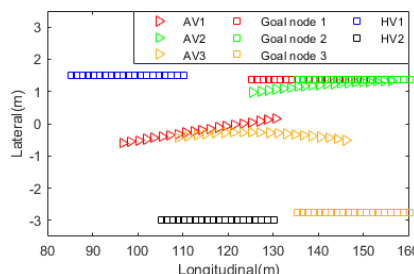

(d) $6-8 \mathrm{~s}$

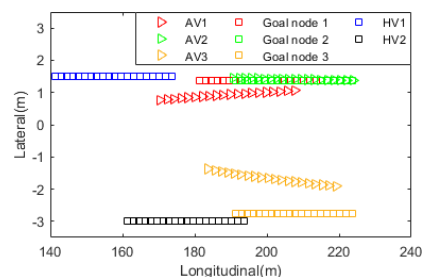

(f) $10-12 \mathrm{~s}$
Fig. 5. The process of cooperative obstacle avoidance.

Fig. 5 depicts the cooperative obstacle avoidance trajectory of each AV fleet member during overtaking. As can be seen from Fig. 5(a), from 0-2s, AV1 and AV2 start to change from lane 1 to lane 2, and AV3 starts to change from lane 2 to lane 1. In Fig. 5(b), the movement trajectory of AV1 shows that AV1 decelerates significantly in the lateral direction, because AV1 is actively avoiding HV1. A more obvious obstacle avoidance behavior appears in Fig. 5(c) and (d), in order to avoid the collision with HV2, AV3 changes from lane 1 to lane 2 , then from lane 2 to lane 1 , and finally completes the overtaking. In Fig. 5(e) and (f), each AV fleet member moves to the target lane and tracks the target position in their own lane. The position relationship between vehicles can be shown more intuitively in Fig. 6 . When $t=1 \mathrm{~s}$, the AV fleet members are still in their initial lane, but they are all making lane changes. When $\mathrm{t}=1.8 \mathrm{~s}, \mathrm{AV} 3$ has already 


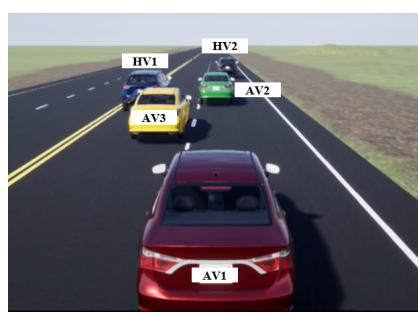

(a) $\mathrm{T}=1 \mathrm{~s}$

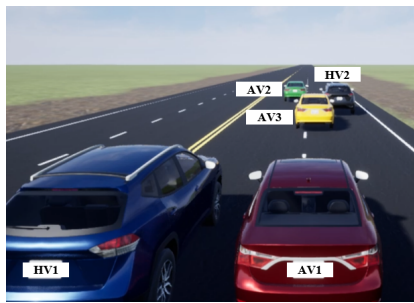

(c) $\mathrm{T}=2.7 \mathrm{~s}$

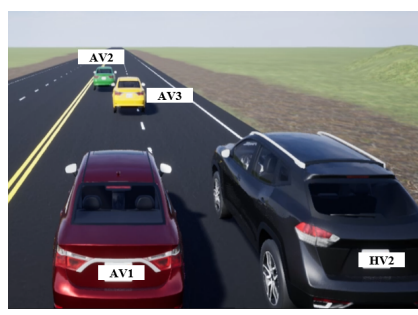

(e) $\mathrm{T}=5.4 \mathrm{~s}$

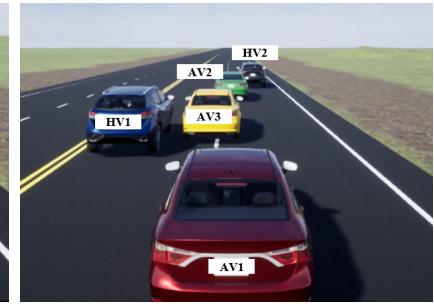

(b) $\mathrm{T}=1.8 \mathrm{~s}$

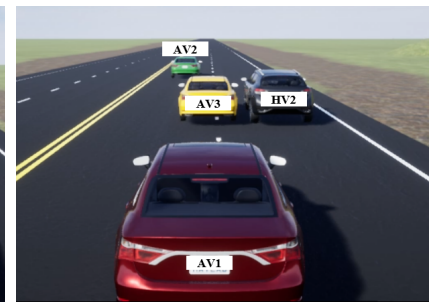

(d) $\mathrm{T}=3.5 \mathrm{~s}$

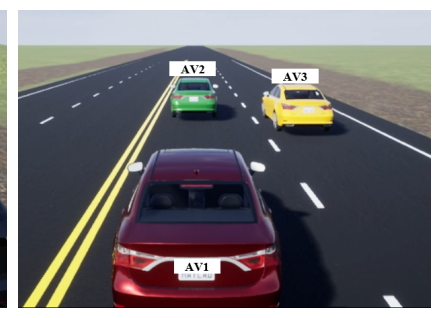

(f) $\mathrm{T}=9 \mathrm{~s}$
Fig. 6. The process of cooperative obstacle avoidance in Unreal Engine.

avoided HV1 and started to overtake, and AV1 and AV2 are about to enter the second lane. As shown in Fig. 6(c), AV2 has already overtaken HV2, and AV3 is about to overtake HV1 by switching from lane 1 to lane 2 . By the time $t=3.5$ s, AV1 has overtaken HV1, AV2 has successfully changed to lane 2, and AV3 has moved to the left and left enough space for overtaking HV2. When AV1 overtakes HV2, the movement state of the whole AV fleet is shown in Fig. 6(f). After that, each AV fleet member will remain in the current lane and accurately track the target position.

The virtual node tracking performance is shown in Fig. 7, where only responses of AV1 are depicted for simplicity. Obviously, the longitudinal and lateral displacement of AV1 eventually converges to the position corresponding to the virtual target node. In Fig. 7(c) and (d), both longitudinal and lateral velocities of AV1 change dramatically due to the need for fast coordinated obstacle avoidance during overtaking. In this case study, three autonomous vehicles have different initial velocities, and after overtaking, the $\mathrm{AV}$ fleet needs to maintain the same velocity. Fig. 8 shows that the velocity of each $\mathrm{AV}$ fleet member converges to a common value. In the first half of the trajectory, the velocity of each AV fleet members did not have an obvious tendency to converge, because the vehicles avoiding obstacles were mainly influenced by the repulsive forces of the neighboring vehicles. In the second half of the trajectory, after the vehicle

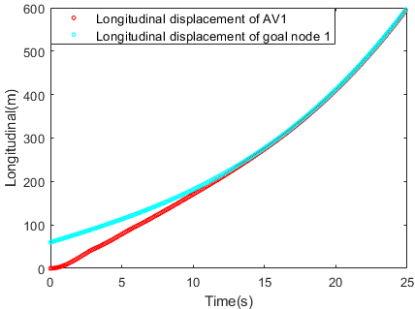

(a) Longitudinal displacement

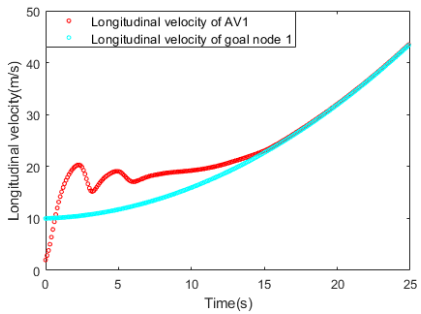

(c) Longitudinal velocity (b) Lateral displacement

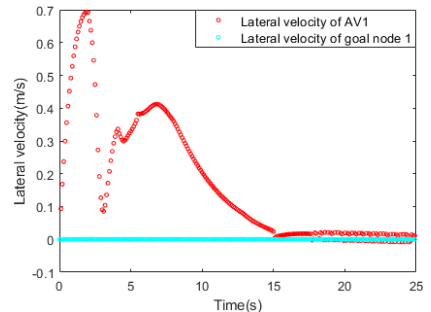

(d) Lateral velocity

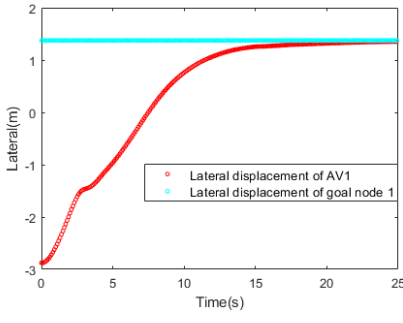

Fig. 7. AV1 tracks the virtual target node. Goal node 1 represents the virtual target mode of AV1.

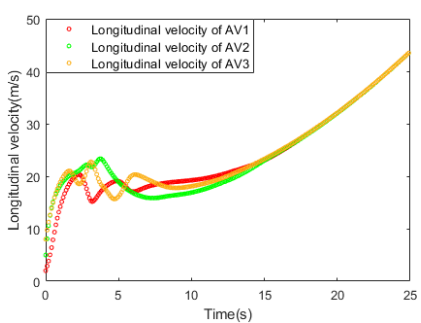

(a) Longitudinal displacement

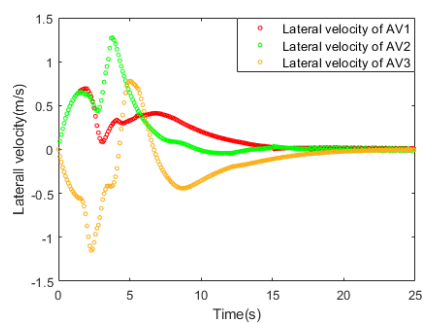

(b) Lateral displacement
Fig. 8. The velocity of each AV fleet member converges to the expected value

completed overtaking and obstacle avoidance, the vehicle was mainly attracted by the virtual target node, so its velocity rapidly converged to the desired value.

Furthermore, we consider using three random initial velocity combinations to test the robustness of the algorithm. The initial velocity values are shown as the Table II. The simulation result in Fig. 9 shows that despite changing the initial speed of the fleet, the AV fleet members still converge to the desired values. Different initial velocity combinations will cause obvious changes in vehicle trajectories during obstacle avoidance and lane change. These facts indicates that the proposed distributed control protocol is robust to the initial velocities of the vehicles.

TABLE II

RANDOM INITIAL VELOCITY COMBINATIONS

\begin{tabular}{ll}
\hline Simulation parameters & Value \\
\hline Initial velocity of AV1,AV2, AV3 in $1^{\text {st }}$ group & $(2,5,8) \mathrm{m} / \mathrm{s}$ \\
Initial velocity of AV1,AV2, AV3 in $2^{\text {nd }}$ group & $(6,5,1) \mathrm{m} / \mathrm{s}$ \\
Initial velocity of AV1,AV2, AV3 in $3^{\text {rd }}$ group & $(7,2,3) \mathrm{m} / \mathrm{s}$ \\
\hline
\end{tabular}




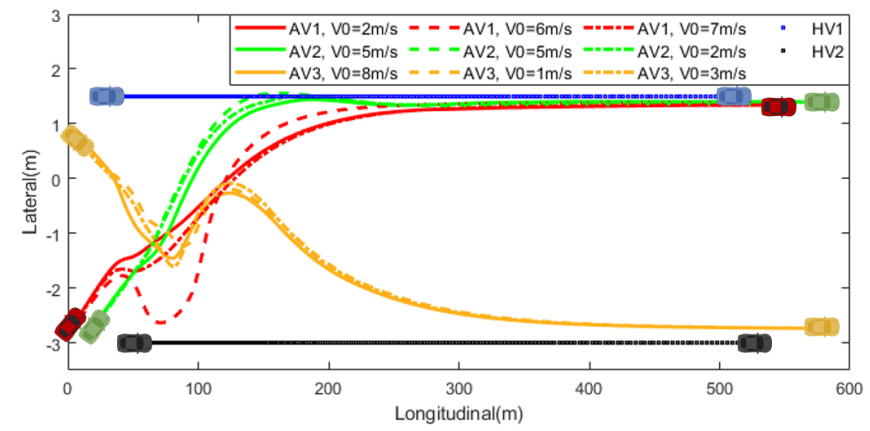

Fig. 9. Overtaking and obstacle avoidance trajectories of AV fleet members at different initial velocities. V0 denotes the initial velocity.

\section{CONCLUSION}

This paper presented a distributed control method for automatic overtaking of autonomous vehicles fleet in a dynamic environment. The simulation results in MATLAB and Unreal Engine proved that the proposed method was accurate and effective for automatic overtaking in the complex dynamic environment with human-driven vehicles. In this paper, the overtaking problem of multi-vehicle system was transformed into multiple dynamic targets' tracking problem. Based on an improved artificial potential field, we constructed a dynamic target tracking control protocol for an AV fleet. A distributed obstacle avoidance control protocol based on artificial potential field was also designed. The simulation results showed that the autonomous vehicle fleet can safely complete overtaking, lane change, obstacle avoidance, and dynamic target tracking.

For future works, the stability of the multi-vehicle system using the proposed method will be mathematically proved. We also need to prove that under the action of this distributed control law, each vehicle $i$ will converge to the virtual target node and will not enter any other local steady states. Besides, the constraints of more realistic vehicle models, such as kinematic constraint and bounded control input, should be considered in the control system design.

\section{ACKNOWLEDGEMENT}

This work was supported by EU H2020-FET-OPEN RoboRoyale project [grant number 964492] and the Engineering and Physical Sciences Research Council (EPSRC) [grant numbers EP/R026084/1 and EP/P01366X/1].

\section{REFERENCES}

[1] W. H. Organization et al., "Global status report on road safety 2018: Summary," World Health Organization, Tech. Rep., 2018.

[2] U. Montanaro, S. Dixit, S. Fallah, M. Dianati, A. Stevens, D. Oxtoby, and A. Mouzakitis, "Towards connected autonomous driving: review of use-cases," Vehicle System Dynamics, vol. 57, no. 6, pp. 779-814, 2019.

[3] W. Payre, J. Cestac, and P. Delhomme, "Intention to use a fully automated car: Attitudes and a priori acceptability," Transportation Research part F: Traffic Psychology and Behaviour, vol. 27, pp. 252 263, 2014.

[4] D. Rojas-Rueda, M. J. Nieuwenhuijsen, H. Khreis, and H. Frumkin, "Autonomous vehicles and public health," Annual Review of Public Health, vol. 41, pp. 329-345, 2020.
[5] J. Hu, P. Bhowmick, F. Arvin, A. Lanzon, and B. Lennox, "Cooperative control of heterogeneous connected vehicle platoons: An adaptive leader-following approach," IEEE Robotics and Automation Letters, vol. 5, no. 2, pp. 977-984, 2020.

[6] K. Zhang, D. Zhang, A. de La Fortelle, X. Wu, and J. Gregoire, "State-driven priority scheduling mechanisms for driverless vehicles approaching intersections," IEEE Transactions on Intelligent Transportation Systems, vol. 16, no. 5, pp. 2487-2500, 2015.

[7] J. Hu, P. Bhowmick, and A. Lanzon, "Group coordinated control of networked mobile robots with applications to object transportation,' IEEE Transactions on Vehicular Technology, vol. 70, no. 8, pp. 82698274, 2021.

[8] D. González, J. Pérez, V. Milanés, and F. Nashashibi, "A review of motion planning techniques for automated vehicles," IEEE Transactions on Intelligent Transportation Systems, vol. 17, no. 4, pp. 1135-1145, 2015.

[9] J. Jiang and A. Astolfi, "Lateral control of an autonomous vehicle," IEEE Transactions on Intelligent Vehicles, vol. 3, no. 2, pp. 228-237, 2018

[10] H. Zhu, K.-V. Yuen, L. Mihaylova, and H. Leung, "Overview of environment perception for intelligent vehicles," IEEE Transactions on Intelligent Transportation Systems, vol. 18, no. 10, pp. 2584-2601, 2017.

[11] J. Hu, P. Bhowmick, I. Jang, F. Arvin, and A. Lanzon, "A decentralized cluster formation containment framework for multirobot systems," IEEE Transactions on Robotics, 2021.

[12] Y. Liu and R. Bucknall, "A survey of formation control and motion planning of multiple unmanned vehicles," Robotica, vol. 36, no. 7, pp. 1019-1047, 2018.

[13] J. Chen, K. Wang, H. Bao, and T. Chen, "A design of cooperative overtaking based on complex lane detection and collision risk estimation," Ieee Access, vol. 7, pp. 87 951-87959, 2019.

[14] N. Wang, X. Wang, P. Palacharla, and T. Ikeuchi, "Cooperative autonomous driving for traffic congestion avoidance through vehicleto-vehicle communications," in 2017 IEEE Vehicular Networking Conference (VNC). IEEE, 2017, pp. 327-330.

[15] J. Hu, A. E. Turgut, B. Lennox, and F. Arvin, "Robust formation coordination of robot swarms with nonlinear dynamics and unknown disturbances: Design and experiments," IEEE Transactions on Circuits and Systems II: Express Briefs, 2021.

[16] B. Wu, L. Qian, M. Lu, D. Qiu, and H. Liang, "Optimal control problem of multi-vehicle cooperative autonomous parking trajectory planning in a connected vehicle environment," IET Intelligent Transport Systems, vol. 13, no. 11, pp. 1677-1685, 2019.

[17] F. Bellotti, S.-W. Kim, and F.-L. Lian, "Introduction to the special issue on applications and systems for collaborative driving," IEEE Transactions on Intelligent Transportation Systems, vol. 18, no. 12, pp. 3457-3460, 2017.

[18] T. Hegedûs, B. Németh, and P. Gáspár, "Graph-based multi-vehicle overtaking strategy for autonomous vehicles," IFAC-PapersOnLine, vol. 52, no. 5, pp. 372-377, 2019.

[19] B. Németh, T. Hegedús, and P. Gáspár, "Model predictive control design for overtaking maneuvers for multi-vehicle scenarios," in 2019 18th European Control Conference (ECC). IEEE, 2019, pp. 744-749.

[20] Í. B. Viana, H. Kanchwala, and N. Aouf, "Cooperative trajectory planning for autonomous driving using nonlinear model predictive control," in 2019 IEEE International Conference on Connected Vehicles and Expo (ICCVE). IEEE, 2019, pp. 1-6.

[21] Í. B. Viana and N. Aouf, "Distributed cooperative path-planning for autonomous vehicles integrating human driver trajectories," in 2018 International Conference on Intelligent Systems (IS). IEEE, 2018, pp. 655-661.

[22] M. Kneissl, A. K. Madhusudhanan, A. Molin, H. Esen, and S. Hirche, "A multi-vehicle control framework with application to automated valet parking," IEEE Transactions on Intelligent Transportation Systems, 2020.

[23] J. Karlsson, C.-I. Vasile, J. Tumova, S. Karaman, and D. Rus, "Multivehicle motion planning for social optimal mobility-on-demand," in 2018 IEEE International Conference on Robotics and Automation (ICRA). IEEE, 2018, pp. 7298-7305.

[24] J. Hu, A. E. Turgut, T. Krajník, B. Lennox, and F. Arvin, "Occlusionbased coordination protocol design for autonomous robotic shepherding tasks," IEEE Transactions on Cognitive and Developmental Systems, 2020. 